\title{
A Mechanism for Polar Protein Localization in Bacteria
}

\author{
Martin Howard \\ Department of Mathematics, Imperial College London, \\ South Kensington Campus, London SW7 2AZ, U.K.
}

(Dated: October 27, 2018)

\begin{abstract}
We investigate a mechanism for the polar localization of proteins in bacteria. We focus on the MinCD/DivIVA system regulating division site placement in the rod-shaped bacterium Bacillus subtilis. Our model relies on a combination of geometric effects and reaction-diffusion dynamics to direct proteins to both cell poles, where division is then blocked. We discuss similarities and differences with related division models in Escherichia coli and also develop extensions of the model to asymmetric polar protein localization. We propose that our mechanism for polar localization may be employed more widely in bacteria, especially in outgrowing spores, which do not possess any pre-existing polar division apparatus from prior division events.
\end{abstract}

\section{INTRODUCTION}

The polar localization of proteins is a vital component of the sophisticated internal organization of bacteria, and is important for many processes ranging from chemotaxis to accurate cell division [1, 2, 3, 4]. However, how such polar localization is achieved has remained largely unknown. The purpose of this report is to analyze one possible mechanism for localization in a well-studied experimental context: the MinCD/DivIVA system regulating cell division positioning in the Gram-positive bacterium B. subtilis 5, 6, 7, 8, 9, 10, 11, 12. We will then discuss how these ideas might be applied more generally to the generation of polar localization/asymmetry in bacteria.

The dynamics of bacterial cell division have been intensively studied, particularly in B. subtilis 5, 6, 7, 8, 9, 10, 11, 12 and E. coli 13, 14, 15, 16, 17, 18, 19, 20, $21,22,23$, where the polar localization of proteins is known to be crucial. In both cases, the location of the division site is determined by the tubulin-like GTPase FtsZ which assembles into a ring-shaped structure just under the cell membrane at midcell. In $E$. coli accurate targeting of the FtsZ ring to midcell is achieved both by the inhibitory effect of the nucleoids ("nucleoid occlusion") and through the MinCDE proteins [16]. MinC and MinD form a complex on the cell membrane, which undergoes coherent pole-to-pole oscillations, with a period of around a minute, driven by MinE. As a result of the oscillations, the time-averaged MinCD concentration is minimized at midcell and maximized at the cell poles. Since MinC inhibits FtsZ polymerization, the FtsZ ring assembles at midcell where the average MinC concentration is lowest. In this way, through the temporary sequestration of MinCD at the cell poles, the midcell can be accurately targeted for cell division.

Recently, several groups have employed simple reaction-diffusion mathematical models (either deterministic [24, 25, 26] or stochastic 27]) to try to understand the MinCDE dynamics (see also Refs. 28, 29] for related models). The principal result of this modeling effort is that the oscillations are a spontaneous self- organized phenomena arising from an intrinsic instability of the reaction-diffusion dynamics. A vital element of this instability is the varying diffusion constants of the MinCDE proteins: high in the cytoplasm; low when polymerically bound to the membrane. Crucially, the oscillations form spontaneously as a result of this disparity of the diffusion constants and of the nonlinear binding/unbinding interactions between the proteins. As a result, the oscillatory dynamics does not require any preexisting topological markers to distinguish various locations within the cell, as had previously been supposed.

Very recently, improved fluorescent imaging has revealed that the $E$. coli Min proteins polymerize into stable helices on the cytoplasmic membrane 23]. This phenomenon is not explicitly included in any of the existing mathematical models, which may therefore require some amendment. Nevertheless, the models generally agree well with fluorescent imaging experiments, especially in the observation of periodic MinCDE/FtsZ bands in filamentous mutants. These results are very difficult to understand other than as a pattern-forming reactiondiffusion instability. Hence, we believe the essentials of a reaction-diffusion mechanism underpinning the MinCDE oscillations are likely to be correct. Although the formation of the Min proteins into filaments has only been observed in E. coli (and also in vitro 22 ), this observation may have important implications in other bacteria. Already in $B$. subtilis the shape-determining proteins MreB and $\mathrm{Mbl}$ are known to form helical cables that could comprise cytoskeletonal-like elements [2, 30].

Despite possessing two of the three same proteins (MinC and MinD, but not MinE), B. subtilis appears to use a quite different mechanism to achieve central placement of the FtsZ ring in vegetative cells $5,6,67,8,9$, 10, 11, 12. In B. subtilis, MinCD do not oscillate, but instead are localized to both cell poles, where they are anchored by the protein DivIVA (which is structurally unrelated to MinE). Also involved are the (initially) homogeneously distributed proteins ZapA, which promotes 31], and EzrA, which inhibits 32], FtsZ ring assembly. Quite how DivIVA is able to recognize the cell poles 
has remained unresolved and forms the main object of this report. With MinCD localized to the cell poles, the FtsZ ring is again unable to nucleate there. However, it is likely that additional mechanisms are used to accurately locate the FtsZ ring to midcell 33], since the MinCD/DivIVA system functions primarily to block polar division 34]. These additional (and so far uncharacterized) mechanisms may be especially important as nucleoid occlusion is ineffective as a means of positioning in B. subtilis [35]. Despite this, the precision of midcell division is the same in E. coli and B. subtilis [34], even though their regulatory mechanisms differ considerably.

Polar protein localization is not only restricted to positioning of the division site. For example in Caulobacter crescentus, polar asymmetry is exploited to ensure that daughter cells are dissimilar: one a swarmer cell with a polar flagellum, and one a stalked cell with a polar stalk that can adhere to a source of food [1, 4]. In each case, the development of a cell with a polar stalk or flagellum is critically dependent on spatiotemporally controlled protein localization, and on the subsequent proteolysis and release of these components at the appropriate time. Other examples of polar localization include the polar clustering of chemoreceptors, in both $E$. coli [36, 37, 38] and C. crescentus [39], and also the polar localization of the virulence protein IcsA [40], which promotes undirectional actin-based motility of the bacterium Shigella flexneri.

\section{POLAR PROTEIN LOCALIZATION IN $B$. SUBTILIS}

Polar division in vegetative cells of $B$. subtilis is blocked by the presence of membrane localized MinC. MinC is normally a cytoplasmically localized protein, however, in the presence of MinD the two interact strongly and bind to the membrane [8]. Polar localization is facilitated by the action of the protein DivIVA, which acts to anchor MinCD to the poles [7, 8]. Targeting of MinCD to the poles, is dependent on DivIVA, as in filamentous cells lacking DivIVA, MinCD was partly distributed diffusively along the cell [7, 8]. Furthermore the localization and activity of MinD is believed to require ATP binding and possibly hydrolysis [9]. However, MinCD can also (MinC more strongly than MinD) target past/present division locations independently of DivIVA [7, 8]. Similarly in a minD mutant, DivIVA simply localizes to division sites regardless of location, again demonstrating an affinity for some component of the cell division apparatus [7. Thus in wild-type cells, MinCD/DivIVA localize to the poles (old division sites), blocking cell division there. However, before septal constriction occurs and the cell divides, MinCD/DivIVA assemble approximately simultaneously at midcell [7], and are retained there after division [7, 8]. In this way MinCD is delivered to the new poles of the two daughter cells, meaning that polar division is immediately prevented at the new poles.

This observation immediately provides one possible explanation for polar localization: since the cell division apparatus marks the location of the new cell poles in the daughter cells, if the relevant proteins can localize to a component of the division apparatus, then the cell pole will continue to be marked in the daughter cells. However, one may question whether this mechanism is really sufficiently robust: if MinCD/DivIVA fail to correctly localize to the site of division, then the poles of the resulting daughter cells will remain unmarked, meaning that division in those cells would be corrupted. It would certainly therefore be desirable to have other means of identifying the cell poles. Experiments in outgrowing spores of $B$. subtilis indicate that this is indeed the case [1, 12]. MinCD/DivIVA are targeted to the cell poles very rapidly after spore germination, even in cells lacking FtsZ. Thus, polar localization can occur even in germinating spores which do not possess pre-existing division apparatus left over from prior division events.

So the question arises, what other mechanism could be responsible for the polar localization? One possible answer is that a self-organized reaction-diffusion instability exists, similar to that already studied in E. coli. Of course, for B. subtilis, the instability would have to be to a steady-state distribution where the MinCD/DivIVA do not oscillate (i.e. in linear stability analysis one would expect a real rather than a complex growth eigenvalue). With appropriate interactions and diffusion constants, such an instability could certainly exist. However, one of the key predictions of such an instability is the existence of a characteristic wavelength, depending on the microscopic parameters of the model, but independent of the geometry [24, 25, 26]. Thus in long filamentous cells of E. coli, several wavelengths can be fit into a single cell and one therefore expects to see multiple oscillating bands. The observation of this phenomena in filamentous $E$. coli [13] is powerful evidence in favor of the reaction-diffusion mechanism. The situation in $B$. subtilis is, however, rather different: in filamentous cells lacking FtsZ, MinCD/DivIVA are primarily localized to the cell poles [7, 8], without any regularly spaced intermediate bands in between, as one would expect for a reaction-diffusion instability. One could argue that the affinity of MinCD/DivIVA for the pre-existing (polar) division apparatus from previous division events might mask the existence of any intermediate bands. However, in outgrowing spores, which have no pre-existing division apparatus, there are still no intermediate bands, even in long filamentous mutants (up to about $13 \mu \mathrm{m}$ long) 11, 12]. Thus we see no evidence for the existence of a characteristic wavelength. Of course, it is just possible that the characteristic wavelength is simply very long, too long to have been seen in past experiments; but, there is currently no hint of this. 
Instead we propose that it is a geometric effect in combination with reaction-diffusion dynamics which marks the end of the cell. The essence of our approach is to assume that MinCD has a reduced rate of membrane binding at the cell poles. If, for example, MinCD polymerize into filaments, as in E. coli [23], this might be due to the increased curvature of the cell poles interfering with membrane polymerization. Since DivIVA may then bind to the edges of MinCD clusters and so stabilize them, the MinCD (and DivIVA) concentrations end up being maximized close to the cell poles, even though MinCD initially has a reduced rate of binding there. Similarly, when the cell divides a region of high curvature is created at midcell, again attracting the MinCD/DivIVA proteins independent of the division apparatus. In the next section, we define this model more precisely and report on the results of numerical simulations.

\section{THE MODEL}

Previous modeling work on the MinCDE system has demonstrated the effectiveness of simple deterministic reaction-diffusion equations [24, 25, 26]. Accordingly we follow a similar modeling approach here. The dynamics of MinC is similar to that of MinD, so, for simplicity, we drop MinC from our model. The fact that MinD can localize normally in $\min C$ mutant cells also supports this approach [8]. Initially, we also assume a "conservative" model, where the MinD/DivIVA are able to bind/unbind from the membrane but are not degraded in the process. This is again similar to some of the models used for $E$. coli [24, 26, 27]. The relevant set of one-dimensional reaction-diffusion equations are:

$$
\begin{aligned}
& \frac{\partial \rho_{D}}{\partial t}=D_{D} \frac{\partial^{2} \rho_{D}}{\partial x^{2}}-\frac{\lambda_{D}(x) \rho_{D}}{\left(1+\lambda_{D}^{\prime 2} \rho_{d}^{2}\right)}+\frac{\lambda_{d} \rho_{d}}{\left(1+\lambda_{d}^{\prime} \rho_{a}\right)} \\
& \frac{\partial \rho_{d}}{\partial t}=D_{d} \frac{\partial^{2} \rho_{d}}{\partial x^{2}}+\frac{\lambda_{D}(x) \rho_{D}}{\left(1+\lambda_{D}^{\prime 2} \rho_{d}^{2}\right)}-\frac{\lambda_{d} \rho_{d}}{\left(1+\lambda_{d}^{\prime} \rho_{a}\right)} \\
& \frac{\partial \rho_{A}}{\partial t}=D_{A} \frac{\partial^{2} \rho_{A}}{\partial x^{2}}-\frac{\lambda_{A} \rho_{A}\left|\frac{\partial \rho_{d}}{\partial x}\right|}{\left(1+\lambda_{A}^{\prime 2} \rho_{a}^{2}\right)}+\frac{\lambda_{a} \rho_{a}}{\left(1+\lambda_{a}^{\prime 2} \rho_{a}^{2}\right)} \\
& \frac{\partial \rho_{a}}{\partial t}=D_{a} \frac{\partial^{2} \rho_{a}}{\partial x^{2}}+\frac{\lambda_{A} \rho_{A}\left|\frac{\partial \rho_{d}}{\partial x}\right|}{\left(1+\lambda_{A}^{\prime 2} \rho_{a}^{2}\right)}-\frac{\lambda_{a} \rho_{a}}{\left(1+\lambda_{a}^{\prime 2} \rho_{a}^{2}\right)} .
\end{aligned}
$$

Here, the first equation is for the cytoplasmic MinD density $\rho_{D}$, the second for the membrane bound MinD density $\rho_{d}$, the third for the cytoplasmic DivIVA density $\rho_{A}$, and the fourth for the membrane bound DivIVA density $\rho_{a}$. In the above model, we assume that MinD binds to the membrane with rate $\lambda_{D}$, in agreement with green fluorescent protein (GFP) fusions which indicate that MinD is a membrane-localized protein 7, 8]. However, one would also expect that the rate of binding is reduced as the MinD membrane density increases. This accounts for the $\lambda_{D}^{\prime}$ term. We also assume that MinD can unbind spontaneously $\left(\lambda_{d}\right.$ term), but that this is suppressed by DivIVA $\left(\lambda_{d}^{\prime}\right.$ term) 7, 8]. It is also possible to insert a factor $\left(1+\lambda_{d}^{\prime \prime} \rho_{d}\right)^{-1}$ in this term, which would model increased stabilization of membrane MinD from clustering. However, we have found that our results were not significantly affected by this term, which we have therefore left out for simplicity. Notice that the DivIVA functions to stabilize the membrane MinCD, hence its role is very different from the $E$. coli MinE protein which destabilizes membrane MinCD. This difference is partly responsible for the structure of the equations being rather different in this case from the earlier models for $E$. coli MinCDE 24, 25, 26. DivIVA is also believed to be an intrinsically cytoplasmic protein 5. Hence, in order to bind to the membrane in outgrowing spores 11], (without preexisting division apparatus), it presumably requires the presence of MinD. We assume here, similar to Ref. [9], that it binds to (and then stabilizes) the edges of MinD clusters. This could correspond to DivIVA "capping" the ends of MinCD filaments [9]. This process would then account for the $\lambda_{A}$ gradient term $\left|\partial \rho_{d} / \partial x\right|$, which is maximized at the edges of a cluster. Note that the gradient term has a modulus sign, as the rate of binding should not depend on the sign of the gradient. Again we assume that the DivIVA binding is suppressed as the membrane DivIVA density increases $\left(\lambda_{A}^{\prime}\right.$ term). Finally DivIVA can spontaneously unbind $\left(\lambda_{a}\right.$ term), but this is suppressed by DivIVA itself ( $\lambda_{a}^{\prime}$ term). This indicates that DivIVA itself oligomerizes/forms clusters for maximum stability, for which there is also experimental evidence [10].

The above assumptions are fairly minimal and are all closely based on the available experimental evidence of GFP fusions and deletion mutants [5, 6, 7, 8, 9, 10, 11, 12. As we have said, we then add one extra ingredient, namely a position dependent membrane binding $\lambda_{D}(x)$ for the MinD, where $\lambda_{D}(x)$ is reduced in regions close to the cell ends (see below).

For our computer simulations, we assume hard wall boundary conditions (i.e. $\partial \rho_{i} / \partial x=0, \quad i=\{D, d, A, a\}$, at the boundaries) and we numerically integrate the above equations, with lattice size $d x=0.02 \mu \mathrm{m}$ and time increment $d t=1 \times 10^{-5} \mathrm{~s}$. We start from random initial conditions, where the MinD and DivIVA are randomly distributed throughout the cytoplasm. We begin the simulation with a total of 50 lattice sites (bacterial length $1 \mu \mathrm{m}$ ), with initial densities of $1000 \mu \mathrm{m}^{-1}$ for MinD and $750 \mu^{-1}$ for DivIVA. The actual concentration levels for B. subtilis have not been accurately measured, so we assume concentration levels similar to those observed in E. coli [41]. During the course of the simulation, we then allow the bacterial length to increase, modeling a growing bacterium, with growth rate of $4 \mu \mathrm{m} /$ hour. In other words, every $1.8 \times 10^{5}$ time steps an extra site is inserted, randomly located. Note, however, that the extra site is never inserted at the inert cell ends [42] (de- 
fined here by regions where $\lambda_{D}(x)$ is reduced, see below). As the bacteria grows in the simulation we add extra MinD/DivIVA, randomly localized in the cytoplasm, to ensure that the average densities remain constant (i.e. the total number of MinD/DivIVA proteins is proportional to the cell length). There is currently no information on whether the average densities do actually remain constant as the cell grows, but the assumption used here is the simplest possible. Nevertheless, precise regulation of the densities turns out not to be particularly important. Considerable overproduction eventually leads to the MinCD/DivIVA proteins saturating the poles and spilling over into the reminder of the cell length. Underproduction simply translates to less MinCD/DivIVA being present along the cell length in between the poles. The important point is that the bacterium has to manufacture sufficient MinCD/DivIVA to ensure that the cell poles are always well populated with these proteins, but not so much that the proteins spill over to midcell leading to division inhibition. Note that in our simulations, we exclusively model the case of outgrowing spores without pre-existing division apparatus. Clearly, modeling protein localization to pre-existing binding sites in wildtype vegetative $B$. subtilis cells is straightforward, and not particularly revealing.

In our simulations we use the following parameter set: $D_{D}=2.0 \mu \mathrm{m}^{2} \mathrm{~s}^{-1}, D_{d}=0, D_{A}=2.0 \mu \mathrm{m}^{2} \mathrm{~s}^{-1}$, $D_{a}=2 \times 10^{-4} \mu \mathrm{m}^{2} \mathrm{~s}^{-1}, \lambda_{D}=10 \mathrm{~s}^{-1}, \lambda_{D}^{\prime}=0.001 \mu \mathrm{m}$, $\lambda_{d}=3.0 \mathrm{~s}^{-1}, \lambda_{d}^{\prime}=0.2 \mu \mathrm{m}, \lambda_{A}=0.03 \mu \mathrm{m}^{2} \mathrm{~s}^{-1}$, $\lambda_{A}^{\prime}=8 \times 10^{-4} \mu m, \lambda_{a}=2.0 s^{-1}, \lambda_{a}^{\prime}=0.005 \mu m$. Furthermore, for the closest 10 sites to both ends of the bacteria, the binding rate $\lambda_{D}$ is reduced by a factor of 2 (our results are robust to changes in this suppression factor). It is also possible to generalize the model so that the MinD/DivIVA proteins are degraded at a (slow) rate, whilst increasing the production rate to keep the average densities constant. We have verified that this modification also makes no qualitative difference to our results. The values for the above cytoplasmic diffusion constants are of the same order as that measured (for different proteins) in the cytoplasm of E. coli [4]]. As for the membrane diffusion constants, we have used similarly small values to those used in E. coli 24, 27]. Previously this was an important component in the spontaneous reaction-diffusion instability; this is, however, not the case here: the small membrane diffusivities are now necessary to preserve the polar density concentration maxima and to prevent the concentrations from equalizing everywhere along the cell membrane. For the remaining parameters there is no experimental guidance, but we have taken care to check that our results are robust to variations in these parameters.

\section{RESULTS: POLAR PROTEIN LOCALIZATION}

In Figure 1, we show the result of our computer simulation of the spore outgrowth experiment. We see very similar results to those observed experimentally [1, 12]. Starting from random initial conditions, the MinD/DivIVA rapidly (within a few minutes) localize to the poles of the cell. This agrees with experiments indicating that MinD/DivIVA localize to the cell poles very early in spore germination [11, 12]. In our model the mechanism for this localization follows from the reduced rate of MinD binding to the cell ends. This reduced polar value for $\lambda_{D}(x)$ is absolutely critical for polar localization as it sets up a pronounced membrane MinD density gradient close to the cell poles. The DivIVA then binds to these positions of increased gradient, stabilizing the MinD/DivIVA. In this way the MinD/DivIVA concentrations end up being maximized close to the cell ends. In fact, the concentrations are not maximized precisely at the poles, where there is actually a dip (see Figures 2 and 3). However the polar concentrations still end up being much higher than the densities well away from the cell ends. As the cell continues to grow, the polar regions become saturated with MinD/DivIVA and the newly produced proteins cannot localize there. Instead the proteins localize to random membrane patches along the cell length. Furthermore, these patches appear to propagate along the cell length (see Figure 1). This movement results from the coupling of the DivIVA to the edges of MinD clusters, slightly away from the local MinD density maxima. Since MinD is stabilized by the DivIVA this encourages MinD to rebind slightly away from its current density maxima. This in turn shifts the position of the maximum MinD density gradient, thus also encouraging the DivIVA to rebind in a shifted location. In this way the coupled MinD/DivIVA density maxima both tend to propagate along the membrane, as shown in Figure 1. It would be interesting to see if such movement can be observed in experiments. A snapshot of these patterns can be seen in Figure 2, where we show the MinD/DivIVA membrane density profiles at length $10 \mu \mathrm{m}$. The DivIVA pattern is very similar to that observed experimentally in filamentous spore outgrowth experiments 11, 12], with sharp polar peaks and less intense randomly distributed membrane patches along the cell length. Note that this disordered pattern is seen everywhere inside the simulated outgrowing spore (including close to the cell ends) if $\lambda_{D}(x)$ is a constant, emphasizing again that polar protein localization is critically dependent on a positiondependent MinD binding rate.

More quantitative data, with profiles of the membrane MinD/DivIVA densities at different times are shown in Figure 3(a,c,e). In Figure 3(b,d,f) we show examples of the membrane density averaged over $90 s$ as a function of position for various total cell lengths. A $90 s$ average is 


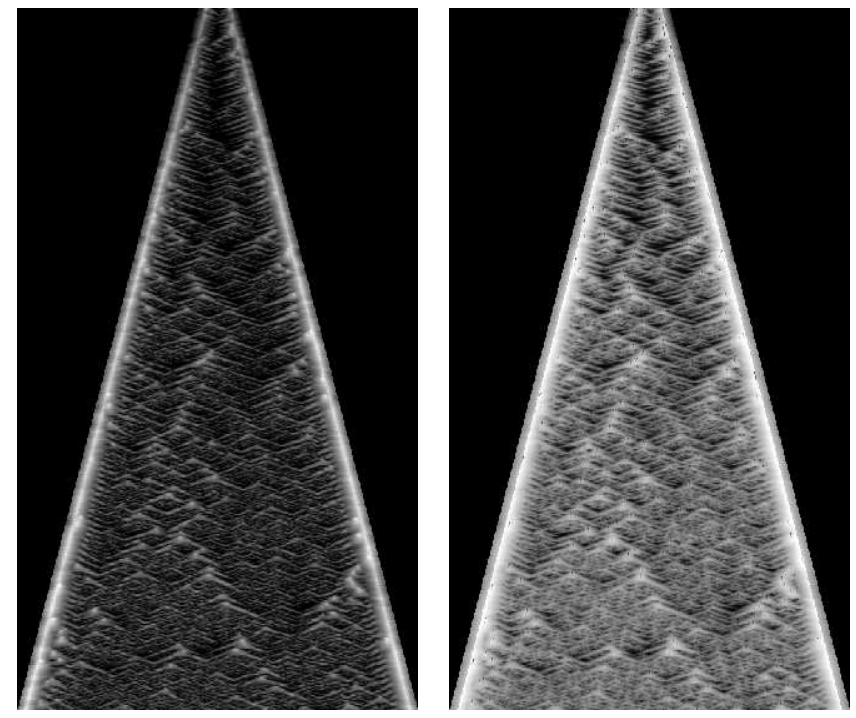

FIG. 1: Spacetime plot of membrane--bound DivIVA (left) and MinD (right) densities in a simulated outgrowing spore. Time increases from top to bottom and the simulation lasts $8100 \mathrm{~s}$, starting with length $1 \mu \mathrm{m}$ and ending with length $10 \mu \mathrm{m}$. White (black) corresponds to areas of high (low) concentration.

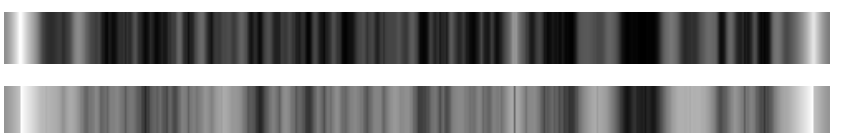

FIG. 2: Snapshot of the DivIVA (top) and MinD (bottom) membrane density profiles at length $10 \mu \mathrm{m}$. White (black) corresponds to areas of high (low) concentration. Note that these are one dimensional simulation results (two dimensional presentation in the figure is for clarity).

chosen as, at least in E. coli, this is the rough time over which the FtsZ averages in locating the FtsZ ring 13]. As can be seen, the MinD concentration is nicely minimized at the cell center for shorter cell lengths. However, as the cell length increases, the precision with which the center can be identified is considerably reduced, with the averaged signal fluctuating considerably at $8 \mu \mathrm{m}$. This data is consistent with evidence that the MinCD/DivIVA system is primarily responsible for blocking polar division, rather than in accurately placing midcell division [34]. Notice also how the MinD is less tightly localized at the cell poles; its signal decreases more slowly than for the DivIVA towards the center of the cell. This also agrees with experiment [7].

\section{RESULTS: ASYMMETRIC PROTEIN LOCALIZATION}

Up to this point, we have modeled symmetric protein localization, such as that seen in the MinCD/DivIVA system in B. subtilis. However, it is also interesting to ask (a)

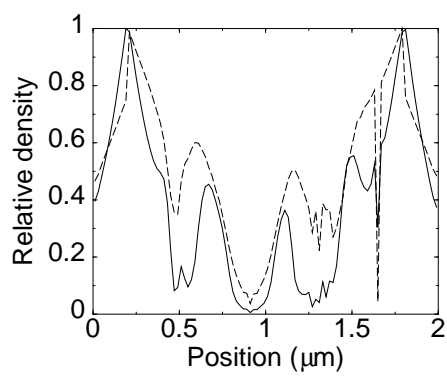

(c)

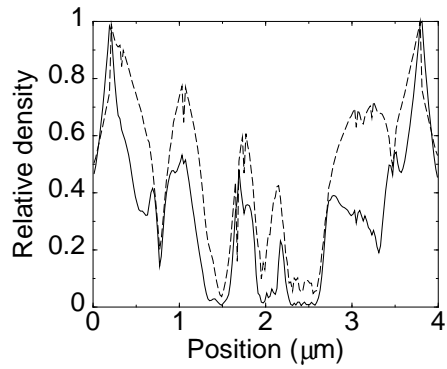

(e)

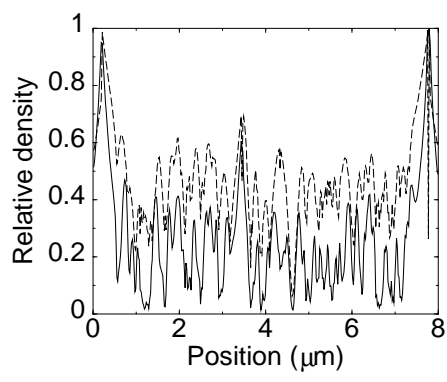

(b)

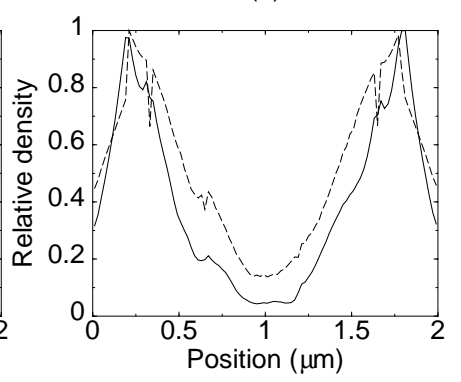

(d)

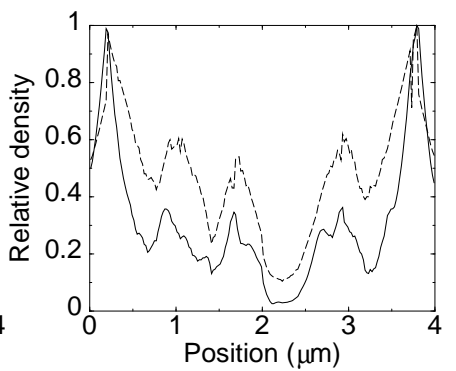

(f)

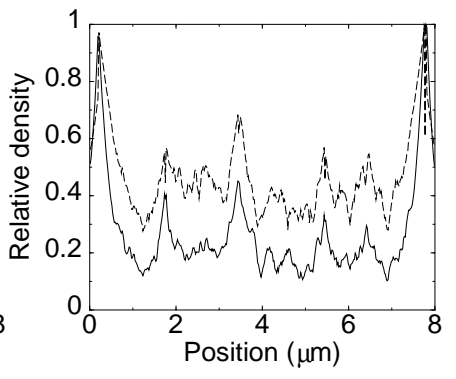

FIG. 3: (a,c,e) Simulations of outgrowing spores: instantaneous membrane MinD (dashed line), and membrane DivIVA (full line) density profiles at lengths $2 \mu \mathrm{m}, 4 \mu \mathrm{m}$ and $8 \mu \mathrm{m}$; (b,d,f) Membrane MinD (dashed line) and membrane DivIVA (full line) density profiles averaged over $90 \mathrm{~s}$ at lengths $2 \mu \mathrm{m}$, $4 \mu \mathrm{m}$ and $8 \mu \mathrm{m}$. Maximum densities for each of the membrane MinD and DivIVA normalized to unity.

whether one can modify the model to obtain asymmetric localization, i.e. to one pole only. This modification turns out to be quite straightforward: in essence one merely has to remove the $\lambda_{D}^{\prime}$ and $\lambda_{A}^{\prime}$ terms which restrict membrane binding when the membrane densities are already high. In this case, starting from random initial conditions, the dynamics rapidly localizes the proteins to both cell poles. However, one randomly chosen pole will, due to fluctuations in the random initial conditions, gain a slightly higher density than the other. Since higher densities yield greater stability for the membrane aggregations (due to the $\lambda_{d}^{\prime}$ and $\lambda_{a}^{\prime}$ terms), and since there are no longer saturation effects, the favored pole, with an initially slightly higher concentration, will continue to attract additional proteins from the other pole, via cytoplasmic diffusion and then polar membrane capture (cf. Ref. [44]). Of course this process will also occur in reverse from the favored to the 
less favored pole, but because of its higher density, the net rate of accumulation will be towards the pole with the higher initial concentration. In this way, the less favored pole is eventually drained of its protein population. Starting from randomly distributed cytoplasmic MinD and DivIVA, with average densities $1000 \mu \mathrm{m}^{-1}$, and $750 \mu \mathrm{m}^{-1}$, respectively, an appropriate parameter set is: $D_{D}=2.0 \mu \mathrm{m}^{2} \mathrm{~s}^{-1}, D_{d}=0, D_{A}=2.0 \mu \mathrm{m}^{2} \mathrm{~s}^{-1}, D_{a}=$ $2 \times 10^{-4} \mu \mathrm{m}^{2} \mathrm{~s}^{-1}, \lambda_{D}=10 \mathrm{~s}^{-1}, \lambda_{D}^{\prime}=0, \lambda_{d}=3.0 \mathrm{~s}^{-1}$, $\lambda_{d}^{\prime}=0.001 \mu \mathrm{m}, \lambda_{A}=0.012 \mu \mathrm{m}^{2} \mathrm{~s}^{-1}, \lambda_{A}^{\prime}=0, \lambda_{a}=$ $1.0 \mathrm{~s}^{-1}, \lambda_{a}^{\prime}=1.2 \times 10^{-4} \mu \mathrm{m}$. In this case localization to a (randomly selected) pole occurs in about $800 \mathrm{~s}$ (data not shown). Alternatively, replacing some of the above parameters with $\lambda_{d}^{\prime}=1 \times 10^{-4} \mu \mathrm{m}, \lambda_{A}=0.002 \mu \mathrm{m}^{2} \mathrm{~s}^{-1}$, $\lambda_{a}=1.6 \mathrm{~s}^{-1}$, and $\lambda_{a}^{\prime}=1.6 \times 10^{-4} \mu \mathrm{m}$, unipolar localization occurs far more rapidly, within about $40 \mathrm{~s}$. A process analogous to this may be happening in asymmetric division in sporulating B. subtilis [45] (see below), although in that case the protein SpoIIE may also play a crucial role [46].

\section{DISCUSSION}

In this paper, we have proposed a mechanism for the symmetric or asymmetric polar localization of proteins, relying on a combination of geometric information and reaction-diffusion dynamics. This mechanism could be particularly important for polar protein localization in cells growing out from spores, where there are no preexisting division apparatus from prior division events. However, even in vegetative cells, where the poles could be marked by the division apparatus from prior divisions, the proposed mechanism could be useful for increased reliability (and redundancy) in locating the poles. We have focused particular attention on the MinCD/DivIVA system in B. subtilis, where our results agree well with experiments on outgrowing spores. We have also made a number of testable assumptions: very low diffusion constants for membrane-bound MinCD/DivIVA; DivIVA binds to the edges of MinD clusters; and membrane binding of MinD is heavily influenced by geometric effects (possibly by the high curvature of the cell poles restricting MinD membrane polymerization). We should point out, however, that experiments in round cell mutants have indicated that simple curvature of the cell membrane is insufficient to elicit DivIVA targeting [47]. Nevertheless, this does not contradict our results: according to our model, what is important is that there be a difference in curvature: one part of the cell with low curvature, another part (the cell poles) with high curvature.

As we mentioned in the introduction there are several other regulatory systems in bacteria with similar polar localization properties. For example, the MCP chemotaxis receptor proteins (in E. coli) 36, 37, 38] and McpA (in C. crescentus) [39] are both polar localized. Fur- thermore, filamentous mutants in both cases show an intriguing pattern of localization: the proteins are still polar localized, but in addition there is a seemingly randomly distributed component along the filament length between the two poles. This pattern is qualitatively reminiscent of the pattern seen (and modeled in this paper) for MinCD/DivIVA in filamentous B. subtilis mutants grown from spores [11, 12]. Although, for the chemotaxis cases, it may simply be that the proteins have saturated polar binding sites and are then "spilling" over into the remainder of the cell. However it is again possible that there may be a geometric component to the localization, similar to that proposed in this report. This may be even more likely in the case of chemotactic proteins in outgrowing spores. Already the chemotaxis protein McpB is known to be polar localized in vegetative B. subtilis [48]. It would be very interesting to study its localization properties in outgrowing spores without pre-existing division apparatus. In addition, it has been shown that DivIVA localizes to division sites both in $E$. coli and, unexpectedly, in the fission yeast Schizosaccharomyces pombe [4]. However, this may again be due to binding to division apparatus proteins (possibly actin in S. pombe).

Given that the geometric properties (high curvature) of the cell poles may be important in influencing MinD binding, it is, of course, important to understand how the cell shape is maintained. In B. subtilis, cell elongation and maintenance of the cell cylinder is achieved by Mbl-directed dispersed helical insertion of cell wall material throughout the cylinder, while the hemispherical cell poles are effectively inert [42]. In vegetative cells, where a midcell septum is formed, it is plausible to see how the hemispherical end is constructed and thus how overall cell shape maintained. However, in spore outgrowth, this is not so obvious. B. subtilis spores themselves are already ellipsoidally shaped, so the initial state for spore germination already has the ends marked via high curvature. However quite how this shape is produced in the earlier sporulation event is rather unclear.

It is also intriguing to note that, although MinC and MinD are used by both B. subtilis and E. coli, the two bacteria appear to use entirely different physical mechanisms to exclude division from polar sites. $E$. coli uses a spontaneous reaction-diffusion instability, whereas geometric effects (reduced polar binding of MinD) and associated reaction-diffusion kinetics, appear to be employed in B. subtilis. Interestingly, it is actually a reduced rate of MinD membrane binding at the cell poles which, in our model, eventually leads to polar localization due to DivIVA binding/stabilizing the edges of MinD membrane clusters. It would be interesting to further study these mechanisms with their evolutionary origin in mind, especially since both employ reaction-diffusion dynamics. Although we believe that the mechanism outlined in this paper provides a very plausible scenario for polar protein localization, other mechanisms are still possible. For ex- 
ample, one could imagine that the DivIVA makes the poles more attractive for MinCD polymerization, perhaps by altering the structure of the MinCD filaments so that they prefer a higher curvature region (see also Ref. 11]). Given that the model described in this paper provides several testable predictions, experiments should be readily able to discriminate between these possible models.

One may also speculate on why E. coli and B. subtilis have differing mechanisms. One possible explanation is that $B$. subtilis must have extra flexibility in its cell division regulation as, unlike $E$. coli, it sporulates when confronted with harsh environmental conditions. In the process of forming a spore, the division septum is relocated from midcell to one of the cell poles. Hence $B$. subtilis must have the ability to undo its polar division block. All that would be required is for another protein to bind to DivIVA, displacing the MinCD, thus freeing up the polar sites, as suggested in Ref. 49]. A suitable candidate for such a displacing protein has recently been shown to be RacA [4]. The MinCD presumably then relocates to the cell center, forcing the FtsZ ring to spiral outwards to the cell poles, as observed in Ref. [45]. As only a single pole is then selected for spore formation, the cytokinetic proteins should localize asymmetrically, possibly in a similar way to that discussed above.

Finally, we mention that the repertoire of division site positioning is likely still not exhausted by the MinCDE and DivIVA systems analyzed here and in previous modeling work [24, 25, 26, 27]. C. crescentus is able to divide asymmetrically with high precision even though it lacks all of the MinCDE and DivIVA proteins [4]. How this is achieved remains unknown and hints that there may be yet other mechanisms capable of providing accurate positional information and hence of regulating subcellular architecture.

\section{Acknowledgements}

This work was supported by The Royal Society.

[1] Shapiro, L., McAdams, H. H. \& Losick, R. (2002). Generating and Exploiting Polarity in Bacteria. Science, 298, 1942-1946.

[2] Errington, J. (2003). Dynamic proteins and a cytoskeleton in bacteria. Nature Cell Biol. 5, 175-178.

[3] Lutkenhaus, J. (2002). Dynamic proteins in bacteria. Curr. Opin. Microbiol. 5, 548-552.

[4] Ryan K. R. \& Shapiro, L. (2003). Temporal and spatial regulation in prokaryotic cell cycle progression and development. Annu. Rev. Biochem. 72, 367-394.

[5] Cha, J.-H. \& Stewart, G. C. (1997). The divIVA Minicell Locus of Bacillus subtilis. J. Bacteriol. 179, 1671-1683.

[6] Edwards, D. H. \& Errington, J. (1997). The Bacillus subtilis DivIVA protein targets to the division septum and controls the site specificity of cell division. Mol. Microbiol. 24, 905-915.

[7] Marston, A. L., Thomaides, H. B., Edwards, D. H., Sharpe, M. E. \& Errington, J. (1998). Polar localization of the MinD protein of Bacillus subtilis and its role in selection of the mid-cell division site. Genes $\&$ Dev. 12, 3419-3430.

[8] Marston, A. L. \& Errington, J. (1999). Selection of the midcell division site in Bacillus subtilis through MinDdependent polar localization and activation of MinC. Mol. Microbiol. 33, 84-96.

[9] El Karoui, M. \& Errington, J. (2001). Isolation and characterization of topological specificity mutants of $\min D$ in Bacillus subtilis. Mol. Microbiol. 42, 1211-1221.

[10] Muchová, K., Kutejová, E., Scott, D. J., Brannigan, J. A., Lewis, R. J., Wilkinson, A. J. \& Barák, I. (2002). Oligomerization of the Bacillus subtilis division protein DivIVA. Microbiology, 148, 807-813.

[11] Harry, E. J. \& Lewis, P. J. (2003). Early targeting of Min proteins to the cell poles in germinated spores of Bacillus subtilis: evidence for division apparatus-independent recruitment of Min proteins to the division site. Mol. Microbiol. 47, 37-48.

[12] Hamoen, L. W. \& Errington, J. (2003). Polar Targeting of DivIVA in Bacillus subtilis Is Not Directly Dependent on FtsZ or PBP 2B. J. Bacteriol. 185, 693-697.

[13] Raskin, D. M. \& de Boer, P. A. J. (1999). Rapid pole-topole oscillation of a protein required for directing division to the middle of Escherichia coli. Proc. Natl. Acad. Sci. USA, 96, 4971-4976.

[14] Raskin, D. M. \& de Boer, P. A. J. (1999). MinDEDependent Pole-to-Pole Oscillation of Division Inhibitor MinC in Escherichia coli. J. Bacteriol. 181, 6419-6424.

[15] Hu, Z. \& Lutkenhaus, J. (1999). Topological regulation of cell division in Escherichia coli involves rapid pole to pole oscillation of the division inhibitor MinC under the control of MinD and MinE. Mol. Microbiol. 34, 82-90.

[16] Yu, X.-C. \& Margolin, W. (1999). FtsZ ring clusters in min and partition mutants: role of both the Min system and the nucleoid in regulating FtsZ ring localization. Mol. Microbiol. 32, 315-326.

[17] Rowland, S. L., Fu, X., Sayed, M. A., Zhang, Y., Cook, W. R. \& Rothfield, L. I. (2000). Membrane Redistribution of the Escherichia coli MinD Protein Induced by MinE. J. Bacteriol. 182, 613-619.

[18] Fu, X., Shih, Y.-L., Zhang, Y. \& Rothfield, L. I. (2001). The MinE ring required for proper placement of the division site is a mobile structure that changes its cellular location during the Escherichia coli division cycle. Proc. Natl. Acad. Sci. USA, 98, 980-985.

[19] Rothfield, L. I., Shih, Y.-L. \& King, G. (2001). Polar Explorers: Membrane Proteins that Determine Division Site Placement. Cell, 106, 13-16.

[20] Hale, C. A., Meinhardt, H. \& de Boer, P. A. J. (2001). Dynamic localization cycle of the cell division regulator MinE in Escherichia coli. EMBO J. 20, 1563-1572.

[21] Hu, Z., Gogol, E. P. \& Lutkenhaus, J. (2002). Dynamic assembly of MinD on phospholipid vesicles regulated by ATP and MinE. Proc. Natl. Acad. Sci. USA, 99, 67616766.

[22] Suefuji, K., Valluzzi, R. \& RayChaudhuri, D. (2002). Dynamic assembly of MinD into filament bundles modulated by ATP, phospholipids, and MinE. Proc. Natl. Acad. Sci. USA, 99, 16776-16781. 
[23] Shih, Y.-L., Le, T. \& Rothfield, L. (2003). Division site selection in Escherichia coli involves dynamic redistribution of Min proteins within coiled structures that extend between the two cell poles. Proc. Natl. Acad. Sci. USA, 100, 7865-7870.

[24] Howard, M., Rutenberg, A. D. \& de Vet, S. (2001). Dynamic Compartmentalization of Bacteria: Accurate Division in E. coli. Phys. Rev. Lett. 87, 278102.

[25] Meinhardt, H. \& de Boer, P. A. J. (2001). Pattern formation in Escherichia coli: A model for the pole-to-pole oscillations of Min proteins and the localization of the division site. Proc. Natl. Acad. Sci. USA, 98, 14202-14207.

[26] Kruse, K. (2002). A Dynamic Model for Determining the Middle of Escherichia coli. Biophys. J. 82, 618-627.

[27] Howard, M. \& Rutenberg, A. D. (2003). Pattern Formation inside Bacteria: Fluctuations due to the Low Copy Number of Proteins. Phys. Rev. Lett. 90, 128102.

[28] Meinhardt, M. (1999). Orientation of chemotactic cells and growth cones: models and mechanisms. J. Cell Sci. 112, 2867-2874.

[29] Hunding, A., Ebersbach, G. \& Gerdes, K. (2003). A Mechanism for ParB-dependent Waves of ParA, a Protein Related to DNA Segregation during Cell Division in Prokaryotes. J. Mol. Biol. 329, 35-43.

[30] Jones, L. J. F., Carballido-López, R. \& Errington, J. (2001). Control of Cell Shape in Bacteria: Helical, Actinlike Filaments in Bacillus subtilis. Cell, 104, 913-922.

[31] Gueiros-Filho, F. J. \& Losick, R. (2002). A widely conserved bacterial cell division protein that promotes assembly of the tubulin-like protein FtsZ. Genes $\&$ Dev. 16, 2544-2556.

[32] Levin, P. A., Kurtser, I. G. \& Grossman, A. D. (1999). Identification and characterization of a negative regulator of FtsZ ring formation in Bacillus subtilis. Proc. Natl. Acad. Sci. USA, 96, 9642-9647.

[33] Levin, P. A., Shim, J. J. \& Grossman, A. D. (1998). Effect of minCD on FtsZ Ring Position and Polar Septation in Bacillus subtilis. J. Bacteriol. 180, 6048-6051.

[34] Migocki, M. D., Freeman, M. K., Wake, R. G. \& Harry, E. J. (2002). The Min system is not required for precise placement of the midcell $\mathrm{Z}$ ring in Bacillus subtilis. EMBO Rep. 3, 1163-1167.

[35] Regamey, A., Harry, E. J. \& Wake, R. G. (2000). Midcell $\mathrm{Z}$ ring assembly in the absence of entry into the elongation phase of the round of replication in bacteria: co-ordinating chromosome replication with cell division. Mol. Microbiol. 38, 423-434.

[36] Maddock, J. R. \& Shapiro, L. (1993). Polar Location of the Chemoreceptor Complex in the Escherichia coli Cell.
Science, 259, 1717-1723.

[37] Lybarger, S. R. \& Maddock, J. R. (2000). Differences in the polar clustering of the high- and low-abundance chemoreceptors of Escherichia coli. Proc. Natl. Acad. Sci. USA, 97, 8057-8062.

[38] Maki, N., Gestwicki, J. E., Lake, E. M., Kiessling, L. L. \& Adler, J. (2000). Motility and Chemotaxis of Filamentous Cells of Escherichia coli. J. Bacteriol. 182, 4337-4342.

[39] Alley, M. R. K. (2001). The highly conserved domain of the Caulobacter McpA chemoreceptor is required for its polar localization. Mol. Microbiol. 40, 1335-1343.

[40] Robbins, J. R., Monack, D., McCallum, S. J., Vegas, A., Pham, E., Goldberg, M. B. \& Theriot, J. A. (2001). The making of a gradient: IcsA (VirG) polarity in Shigella flexneri. Mol. Microbiol. 41, 861-872.

[41] Shih, Y.-L., Fu, X., King, G. F., Le, T. \& Rothfield, L. (2002). Division site placement in E. coli: mutations that prevent formation of the MinE ring lead to loss of the normal midcell arrest of growth of polar MinD membrane domains. EMBO J. 21, 3347-3357.

[42] Daniel, R. A. \& Errington, J. (2003). Control of Cell Morphogenesis in Bacteria: Two Distinct Ways to Make a Rod-Shaped Cell. Cell, 113, 767-776.

[43] Elowitz, M. B., Surette, M. G., Wolf, P.-E., Stock, J. B. \& Leibler, S. (1999). Protein Mobility in the Cytoplasm of Escherichia coli. J. Bacteriol. 181, 197-203.

[44] Rudner, D. Z., Pan, Q. \& Losick, R. M. (2002). Evidence that subcellular localization of a bacterial membrane protein is achieved by diffusion and capture. Proc. Natl. Acad. Sci. USA, 99, 8701-8706.

[45] Ben-Yehuda, S. \& Losick, R. (2002). Asymmetric Cell Division in B. subtilis Involves a Spiral-like Intermediate of the Cytokinetic Protein FtsZ. Cell, 109, 257-266.

[46] Lucet, I., Feucht, A., Yudkin, M. D. \& Errington J. (2000). Direct interaction between the cell division protein FtsZ and the cell differentiation protein SpoIIE. EMBO J. 19, 1467-1475.

[47] Edwards, D. H., Thomaides, H. B. \& Errington, J. (2000). Promiscuous targeting of Bacillus subtilis cell division protein DivIVA to division sites in Escherichia coli and fission yeast. EMBO J. 19, 2719-2727.

[48] Kirby, J. R., Niewold, T. B., Maloy, S. \& Ordal, G. W. (2000). CheB is required for behavioural responses to negative stimuli during chemotaxis in Bacillus subtilis. Mol. Microbiol. 35, 44-57.

[49] Ben-Yehuda, S., Rudner, D. Z. \& Losick, R. (2003). RacA, a Bacterial Protein That Anchors Chromosomes to the Cell Poles. Science, 299, 532-536. 